\title{
A novel DNA methylation panel accurately detects colorectal cancer independently of molecular pathway
}

\author{
Micaela Freitas ${ }^{1}$, Fábio Ferreira ${ }^{1}$, Sónia Carvalho ${ }^{1,2}$, Fernanda Silva², Paula Lopes ${ }^{1,2}$, Luís Antunes ${ }^{3}$, Sofia Salta ${ }^{1}$,
} Francisca Diniz ${ }^{1}$, Lúcio Lara Santos ${ }^{4}$, José Flávio Videira ${ }^{4}$, Rui Henrique ${ }^{1,2,5^{*}+}$ and Carmen Jerónimo ${ }^{1,5^{*}+}$ (])

\begin{abstract}
Background: Colorectal cancer (CRC) is one of the most incident cancers, associated with significant morbidity and mortality, and usually classified into three main molecular pathways: chromosomal instability, microsatellite instability (MSI) and CpG island methylator phenotype (CIMP). Currently, available screening methods are either costly or of limited specificity, impairing global implementation. More cost-effective strategies, including DNA methylation-based tests, might prove advantageous. Although some are already available, its performance is suboptimal, entailing the need for better candidate biomarkers. Herein, we tested whether combined use of APC, IGF2, MGMT, RASSFIA, and SEPT9 promoter methylation might accurately detect CRC irrespective of molecular subtype.
\end{abstract}

Methods: Selected genes were validated using formalin-fixed paraffin-embedded tissues from 214 CRC and 50 non-malignant colorectal mucosae (CRN). Promoter methylation levels were assessed using real-time quantitative methylation-specific PCR. MSI and CIMP status were determined. Molecular data were correlated with standard clinicopathological features. Diagnostic and prognostic performances were evaluated by receiver operator characteristics curve and survival analyses, respectively.

Results: Except for IGF2, promoter methylation levels were significantly higher in CRC compared to CRN. A threegene panel (MGMT, RASSF1A, SEPT9) identified malignancy with 96.6\% sensitivity, $74.0 \%$ specificity and 91.5 positive predictive value (area under the curve: 0.97), independently of tumor location, stage, and molecular pathway.

Conclusions: Combined promoter methylation analysis of MGMT/RASSFIA/SEPT9 displays a better performance than currently available epigenetic-based biomarkers for CRC, providing the basis for the development of a non-invasive assay to detect CRC irrespective of the molecular pathway.

Keywords: Colorectal cancer, Gene promoter methylation, Biomarkers, Diagnosis, Prognosis

\footnotetext{
*Correspondence: rmhenrique@icbas.up.pt;

henrique@ipoporto.min-saude.pt; carmenjeronimo@ipoporto.min-saude.

pt; cljeronimo@icbas.up.pt

${ }^{\dagger}$ Rui Henrique and Carmen Jerónimo Joint senior authors

${ }^{1}$ Cancer Biology \& Epigenetics Group-Research Center (CI-IPOP),

Research Center-LAB 3, Portuguese Oncology Institute of Porto

(IPO Porto), F Bdg, 1st Floor, Rua Dr António Bernardino de Almeida,

4200-072 Porto, Portugal

Full list of author information is available at the end of the article
} 


\section{Background}

Colorectal cancer (CRC) is the third most incident and the fourth leading cause of cancer-related death by cancer, worldwide [1]. The primary molecular pathways involved in CRC carcinogenesis are chromosomal instability (CIN), microsatellite instability (MSI) and CpG island methylator phenotype (CIMP), accounting for nearly 85,15 and $10-40 \%$ of all sporadic cases, respectively [2,3]. CIN and MSI represent two levels of genetic instability, a subtle one affecting only DNA sequences, MSI-high (MSI-H), and a gross one, affecting portions or entire chromosomes, i.e., CIN. These forms of instability are considered mutually exclusive: a CRC with CIN is most likely microsatellite stable (MSS) $[4,5]$. In sporadic $\mathrm{CRC}$, the most common cause of DNA mismatch repair (MMR) defects, leading to MSI-H, is aberrant biallelic $M L H 1$ aberrant promoter methylation. $M L H 1$ is also one of the markers often used to define CIMP, and, thus, an overlap between CIMP and MSI-H exists [2]. Two different panels have frequently been used to define CIMP, the classic panel proposed by An et al. ( $M L H 1$, CDKN2A (p16), MINT1, MINT2, and MINT31) and the panel designed by Weisenberger et al. (CACNA1G, IGF2, NEUROG1, RUNX3, and SOCS1) [6, 7]. Another categorization subdivides tumors into CIMP-High (CIMP-H), CIMP-Low (CIMP-L) and CIMP-negative (CIMP-0), each of them associated with different features [8]. Thus, different panels and/or a number of markers have been tested, and no consensus has been reached.

Several non-CIMP related methylation DNA targets have been found to distinguish malignant from nonmalignant colorectal tissues [9]. Indeed, several studies have been conducted to identify a methylation biomarker or a panel of biomarkers with high sensitivity and specificity to be used in diagnosis and prognostication of CRC, but none has been validated [7, 10-21]. Moreover, only a few DNA methylation biomarkers intended for CRC detection are commercially available, including ColoVantage $^{\circledR}$, EpiproColon ${ }^{\circledR} 2.0$ and Abbott RealTime mS9, which are blood-based tests based on septin 9 (SEPT9) promoter methylation [12, 18]. Nonetheless, the value of SEPT9 promoter methylation as a biomarker has been questioned by several authors [22]. Because currently available screening methods are either costly or of limited specificity, impairing global implementation, DNA methylation-based tests are likely to be more costeffective. Owing to the suboptimal performance of commercially available epigenetic tests, validation of better candidate biomarkers, which may detect CRC irrespective of molecular subtype, is warranted.

Based on an exhaustive literature review to select potentially useful gene promoters with the ability to discriminate malignant from non-malignant colorectal tissues, enabling its future testing in blood samples, we selected four genes hypermethylated in CRC [Adenomatous polyposis coli $(A P C)$ [13, 14], O-6-methylguanine-DNA methyltransferase $(M G M T)[15,23]$, Ras association domain family 1-isoform A (RASSF1A) [16] and Septin 9 (SEPT9) $[12,17,18]]$ and one gene hypomethylated in CRC [Insulin-like growth factor 2 (IGF2) [19-21]], for validation in a large cohort of CRCs, in which CIMP and MSI status were also determined. Furthermore, the potential prognostic value of gene promoter methylation was also assessed.

\section{Methods \\ Patients and samples}

A total of 214 CRC (110 colonic and 104 rectal cancers) from patients consecutively diagnosed and treated with surgical resection between 2000 and 2012 at Portuguese Oncology Institute of Porto (IPO Porto), Portugal, were included in this study (Table 1). Fifty samples of noncancerous colorectal mucosa (CRN) from individuals with no evidence of CRC or other gastrointestinal tract cancer were used as controls (Additional file 1: Table S1). All samples corresponded to formalin-fixed paraffinembedded (FFPE) tissues archived at the Department of Pathology of IPO Porto. Haematoxylin and eosin (H\&E) stained tissue sections were reviewed and classified by an experienced pathologist according to current WHO classification (2010). Representative tumor areas were delimitated for further microdissection. Relevant clinical data were collected from medical charts, and tumor staging was performed using the American Joint Committee on Cancer (AJCC) criteria. This study was approved by the institutional ethics committee (CES 120/015).

\section{Immunohistochemistry}

Assessment of MSI status was accomplished through an immunohistochemical assessment of MLH1, MSH2, MSH6, and PMS2 expression, performed as previously described [24].

\section{Quantitative DNA methylation analysis (qMSP)}

DNA was extracted from FFPE sections that contained at least 70\% neoplastic cells, using phenol-chloroform conventional method as described previously Ramalho-Carvalho et al. [25]. DNA was quantified using NanoDrop ND-1000 ${ }^{\circledR}$ (NanoDrop Technologies, DE, USA) spectrophotometer and modified with sodium bisulfite, using the EZ DNA Methylation-Gold ${ }^{\mathrm{TM}}$ Kit (Zymo Research, Orange, CA, USA) according to manufacturer's instructions. Bisulphite-treated DNA was used as a template for qMSP using specific primers for the target genes $[A P C$, 
Table 1 Clinicopathologic features of CRC patients by tumor location

\begin{tabular}{|c|c|c|c|}
\hline Characteristic & Total $(n=214)$ & Colon $(n=110)$ & Rectum $(n=104)$ \\
\hline Age (years) mean (range) & $60.35(25-80)$ & $60.82(25-80)$ & $59.80(31-80)$ \\
\hline \multicolumn{4}{|l|}{ Gender } \\
\hline Female & $74(34.6 \%)$ & $42(38.2 \%)$ & $32(30.8 \%)$ \\
\hline Male & $140(65.4 \%)$ & $68(61.8 \%)$ & $72(69.2 \%)$ \\
\hline \multicolumn{4}{|l|}{ Stage } \\
\hline$|/| \mid$ & $52(24.3 \%)$ & $24(21.8 \%)$ & $28(26.9 \%)$ \\
\hline III & $52(24.3 \%)$ & $20(18.2 \%)$ & $32(30.8 \%)$ \\
\hline IV & $108(50.5 \%)$ & $64(58.2 \%)$ & $44(42.3 \%)$ \\
\hline Unknown & $2(0.9 \%)$ & $2(1.8 \%)$ & - \\
\hline \multicolumn{4}{|l|}{ Tumor differentiation } \\
\hline Well & $4(1.9 \%)$ & $1(0.9 \%)$ & $3(2.9 \%)$ \\
\hline Moderate & $123(57.5 \%)$ & $79(71.8 \%)$ & $44(42.3 \%)$ \\
\hline Poor & $5(2.3 \%)$ & $4(3.6 \%)$ & $1(1.0 \%)$ \\
\hline Not assessable & $82(38.3 \%)$ & $26(23.6 \%)$ & $56(53.8 \%)$ \\
\hline \multicolumn{4}{|l|}{ KRAS mutation status } \\
\hline Wildtype & $116(54.2 \%)$ & $60(54.5 \%)$ & $56(53.9 \%)$ \\
\hline Mutated & 84 (39.3\%) & $46(41.8 \%)$ & $38(36.5 \%)$ \\
\hline Not available & $14(6.5 \%)$ & $4(3.6 \%)$ & $10(9.6 \%)$ \\
\hline \multicolumn{4}{|l|}{ MSI } \\
\hline MSI-H & $8(3.7 \%)$ & $8(7.3 \%)$ & $0(0 \%)$ \\
\hline MSI-L/MSS & $206(96.3 \%)$ & $102(92.7 \%)$ & $104(100 \%)$ \\
\hline \multicolumn{4}{|l|}{ CIMP } \\
\hline CIMP-positive & $18(8.5 \%)$ & $6(5.6 \%)$ & $12(11.5 \%)$ \\
\hline CIMP-negative & $193(91.5 \%)$ & $101(94.4 \%)$ & $92(88.5 \%)$ \\
\hline \multicolumn{4}{|l|}{ Neoadjuvant treatment } \\
\hline Yes & 69 (32.2\%) & $16(14.5 \%)$ & $53(51.0 \%)$ \\
\hline No & $145(67.8 \%)$ & $94(85.5 \%)$ & $51(49.0 \%)$ \\
\hline \multicolumn{4}{|l|}{ Adjuvant treatment } \\
\hline Yes & $171(80.0 \%)$ & 89 (80.9\%) & $82(78.8 \%)$ \\
\hline No & $45(20.0 \%)$ & $21(19.1 \%)$ & $22(21.2 \%)$ \\
\hline
\end{tabular}

CIMP CpG island methylator phenotype, MSI microsatellite instability, MSI-H MSI high, MSI-L MSI low, MSS microsatellite stable

IGF2, MGMT, RASSF1A and SEPT9 (Additional file 1: Table S2)] and CIMP markers (CDKN2A, MLH1 MINT1, MINT2 and MINT31 [7, 26]). Fluorescence-based realtime PCR assays were performed in 384-well plates in a LightCycler 480 II (Roche, Germany) using KAPA SYBR FAST qPCR Master Mix (Kapa Biosystems, USA). All the samples were run in triplicate and melting curve analysis was performed. Serial dilutions of modified CpGenome ${ }^{\mathrm{TM}}$ Universal Methylated DNA was used to generate a standard curve, and relative methylation levels were calculated as the ratio between the target gene mean quantity and $\beta$-actin, the reference gene, mean quantity, multiplied by 1000 for easier tabulation.

For CIMP status evaluation, each of the five markers composing the classical CIMP panel analyzed was considered methylated if the value of the previously described ratio was higher than any of the ratio values for the selected control samples and more significant than 25th percentile. Tumors were considered CIMP+ when more than one gene promoter was found hypermethylated.

\section{Statistical analysis}

Methylation levels and clinical features were compared within groups using non-parametric tests (Mann-Whitney U test or Kruskall-Wallis test, as appropriate). Clinicopathological variables were compared to CIMP status using Chi square test or Fisher's exact test, as applicable. Diagnostic performance of promoter methylated genes was not assessed in patients who underwent neoadjuvant treatments. ROC curves were constructed for each gene, and the best gene combination was assessed. For each panel were computed the specificity, sensitivity and 
accuracy as well as positive predictive value (PPV) and negative predictive value (NPV) were computed for each panel. The panel was considered positive for a specific sample when at least one of the genes was positive in the individual model.

Survival analysis was performed for disease-free survival (DFS) and disease-specific survival (DSS) through Kaplan-Meier method, and the prognostic significance of clinicopathological variables (age, gender, stage, tumor location, histology, KRAS mutation, MSI, and CIMP status) and methylation biomarkers were assessed using the two-sided Log-rank test to compare survival curves. Methylation levels were categorized using the 25th percentile for IGF2 and the 75th percentile for the other genes. The reference groups considered were non-hypomethylated and non-hypermethylated, respectively. Multivariable analysis was carried out using a Cox proportional hazard model. Statistical analysis was performed using SPSS Statistics 22 (IBM, USA), and graphics were assembled using GraphPad Prism 6 (GraphPad Software, USA). A $P$ value $<0.05$ was considered statistically significant.

\section{Results}

\section{Characteristics of patient population}

Detailed characterization of the patient population is depicted in Table 1. Among the 214 CRC patients enrolled in this study, most were male (65.4\%), and the median age was 60.35 years. Most of the tumors were in the colon (51.4\%) and were at stage IV (50.5\%). Neoadjuvant treatment was performed by $32.2 \%$ of patients, with a predominance of those with rectal location $(51.0 \%$ vs. $14.5 \%$ for colonic location). Nevertheless, a similar proportion of patients with colonic vs. rectal cancer received adjuvant therapy (19.1 and 21.2\%, respectively). Overall, KRAS mutations were identified in $39.3 \%$ of patients.

\section{MSI and CIMP status}

Concerning MSI status, assessed by immunohistochemistry, only eight cases were considered MSI-H (3.7\%), all localized in the colon. Regarding CIMP markers, MINT31 showed the highest methylation frequency, whereas $M L H 1$ displayed the lowest, with 15.2 and $0.9 \%$, respectively. Methylation frequencies of the remaining genes were $11.4 \%$ for CDKN2A, $14.7 \%$ for MINT2 and $6.6 \%$ for MINT1. When methylation of all genes/loci were grouped based on methylation of 0 or one marker versus $>1$ marker for the CIMP phenotype, 18 patients were classified as CIMP-positive (8.5\%), and 193 patients were defined as CIMP-negative (91.5\%).
Gene promoter methylation levels and clinicopathological correlates

APC, MGMT, SEPT9 and RASSF1A methylation levels were significantly higher in CRC compared to normal tissues $(P=0.005, P<0.001, P<0.001$ and $P=0.002$, respectively), whereas IGF2 methylation levels were significantly lower in CRC $(P=0.025)$, as expected (Fig. 1$)$. Although no significant association was found between any of the five promoter methylation levels and patients' age, MGMT methylation levels were significantly higher in female patients compared to males both in the colon $(P=0.048)$ and rectum $(P=0.049)$.

Concerning tumour location, MGMT, SEPT9, and RASSF1A methylation levels were significantly higher in colon cancer (proximal and distal) patients $(P=0.000$, $P=0.000$ and $P=0.002$, respectively), compared to CRN, whereas APC, MGMT and SEPT9 displayed significantly higher promoter methylation levels in rectal cancer $(P=0.018, P=0.0003$ and $P=0.002$, respectively). Moreover, higher SEPT9 methylation levels were found in colon cancer than in rectal cancer patients $(P=0.021)$. Interestingly, for rectal cancer, SEPT9 methylation levels were significantly higher in stage IV than in stages I, II or III $(P=0.001)$, whereas both MGMT and SEPT9 methylation levels were significantly lower in patients that underwent neoadjuvant treatment $(P=0.012$ and $P=0.002$, respectively).

Moreover, and except for $A P C$ promoter methylation levels that were significantly higher in MSI-H tumors $(P=0.012)$, no additional significant differences were found for the remaining genes. Furthermore, no associations were found between gene promoter methylation levels and CIMP status.

\section{Diagnostic performance}

Overall, the best performance was depicted by SEPT9 followed by MGMT (AUCs of 0.950 and 0.894, respectively). Indeed, SEPT9 promoter hypermethylation levels identified CRC with $85.5 \%$ sensitivity, $94.0 \%$ specificity, 97.6\% PPV and 69.1\% NPV. Considering the several combinations of genes in a panel, the best performance was accomplished by MGMT/RASSF1A/SEPT9, with AUC of 0.970, 96.6\% sensitivity, $74.0 \%$ specificity, $91.5 \%$ PPV and 88.1\% NPV (Fig. 2 and Table 2).

Importantly, the MGMT/RASSF1A/SEPT9 panel detect cancer both in the colon (proximal and distal) and rectum with a sensitivity of $95.7 \%$ for colon and $98.0 \%$ for rectum. Furthermore, the panel was also able to identify tumors at any disease stage with similar efficiency (100, 94.2, 95.9\% for stage I/II, stage III and stage IV, respectively), regardless of CIMP and MSI status, further supporting its value for CRC detection (Table 3). 


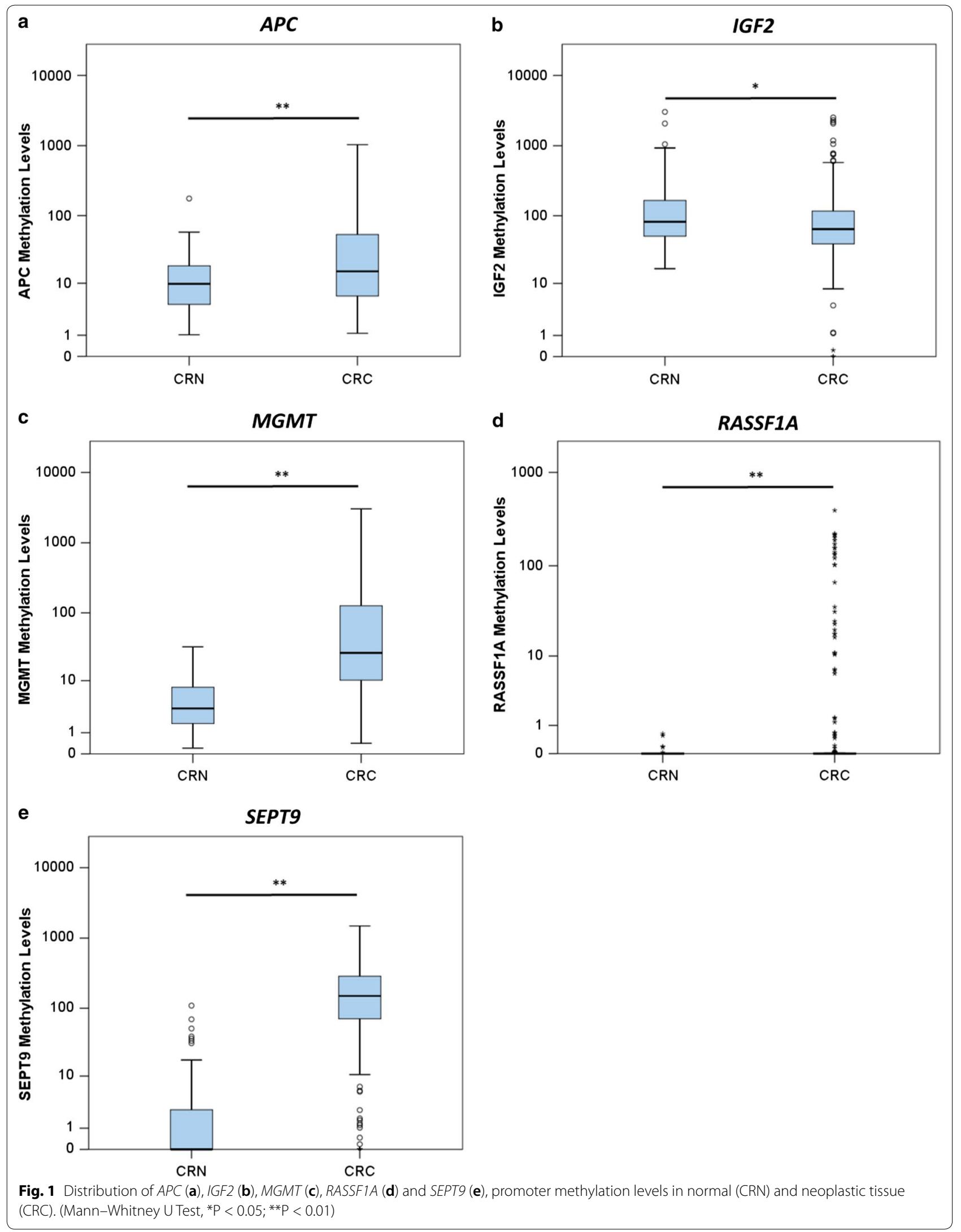




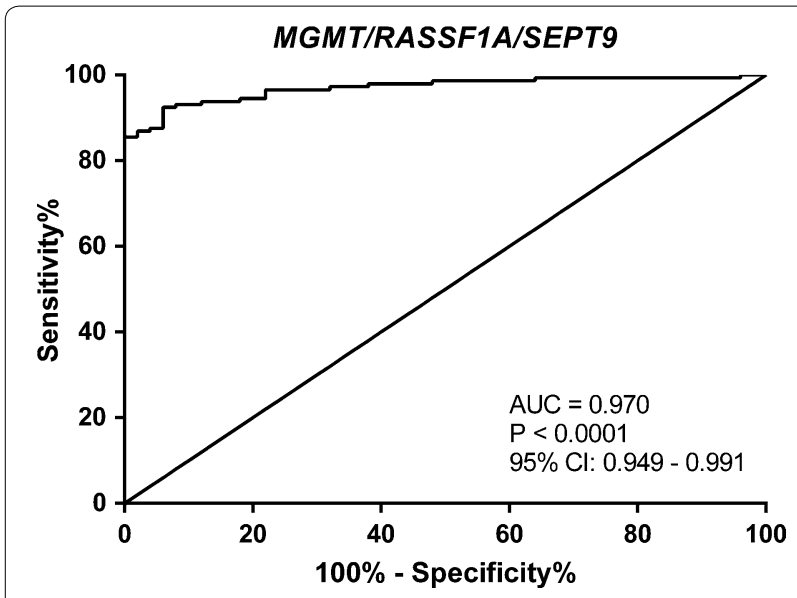

Fig. 2 Receiver operating characteristic curve analysis considering patients without neoadjuvant treatment ( $\mathrm{N}=145$ ) for MGMT/ RASSF1A/SEPT9panel methylation with an AUC of 0.970. AUC, area under curve

\section{Survival analysis}

Considering all CRC cases, SEPT9 promoter methylation levels independently predicted for better DSS [Hazard Ratio $(\mathrm{HR})=0.673,95 \%$ Confidence Interval $(\mathrm{CI})$ 0.469-0.965] while age 60 years or higher and stage IV independently predicted for worse DSS (HR $=1.476$, 95\% CI 1.085-2.008 and HR $=1.862$, 95\% CI 1.295-2.677, respectively). Specifically, in colon cancer, SEPT9 hypermethylation was significantly associated with better prognosis $(\mathrm{HR}=0.472$, 95\% CI 0.276-0.806 and $\mathrm{HR}=0.447$, 95\% CI 0.269-0.744, respectively), whereas age 60 years or higher was associated with worse DSS (HR $=1.730$, 95\% CI 1.079-2.773). Moreover, proximal colon tumors displayed worse prognosis $(\mathrm{HR}=1.879$, 95\% CI $1.174-$ 3.007). On the opposite, in rectal cancer, no associations were found between methylation levels or standard clinicopathological parameters and prognosis (Table 4).

\section{Discussion}

Colorectal cancer is one of the most common and lethal malignancies, especially in developed countries [1]. Currently, CRC screening options mostly rely on fecal occult blood testing and endoscopy. Nevertheless, these strategies meet with significant limitations (e.g., low accuracy and higher cost, respectively) which impair its broader implementation [27]. Some DNA methylation-based biomarkers were developed and have been approved by FDA (Food and Drug Administration), including ColoVantage $^{\circledR}$, Epi proColon ${ }^{\circledR}$, and ColoSure ${ }^{\circledR}$, but are still not fully implemented in clinical practice. Whereas the former two are based on SEPT9 methylation [12, 18], the latter is based on Vimentin methylation [28]. However, SEPT9 promoter methylation performance in CRC detection has been recently questioned [22]. As for Vimentin promoter methylation, this test is used in combination with colonoscopy, but sensitivity is rather variable, ranging from 38 to $88 \%$ [28, 29]. Thus, alternative screening test is demanded to increase population adherence and perfect detection accuracy.

Globally, the distribution of promoter methylation levels for the five gene promoters tested among CRC and CRN tissues paralleled those of previous reports. Indeed, IGF2 methylation levels were significantly lower in CRC than in CRN, in accordance a previous study [19],

Table 3 SEPT9 promoter methylation and 3-gene panel sensitivity values for CRC detection according with location, stage and CIMP and MSI status

\begin{tabular}{lll}
\hline Diagnosis group & SEPT9 & SEPT9, MGMT, RASSF1A \\
\hline Location & & \\
Colon & $84.0(79 / 94)$ & $95.7(90 / 94)$ \\
Proximal & $86.7(26 / 30)$ & $93.3(28 / 30)$ \\
Distal & $82.8(53 / 64)$ & $96.9(62 / 64)$ \\
Rectum & $88.2(45 / 51)$ & $98.0(50 / 51)$ \\
Stage & & \\
Stage I and II & $91.4(32 / 35)$ & $100(35 / 35)$ \\
Stage III & $80.0(28 / 35)$ & $94.2(33 / 35)$ \\
Stage IV & $84.9(62 / 73)$ & $95.9(70 / 73)$ \\
CIMP & & \\
CIMP-negative & $85.5(112 / 131)$ & $96.9(127 / 131)$ \\
CIMP-positive & $90.9(10 / 11)$ & $100(11 / 11)$ \\
MSI & & \\
MSI-L/MSS & $85.4(117 / 137)$ & $97.1(133 / 137)$ \\
MSI-H & $87.5(7 / 8)$ & $87.5(7 / 8)$
\end{tabular}

CIMP CpG island methylator phenotype, MSI microsatellite instability, MSI-H MSI high, MSI-L MSI low, MSS microsatellite stable, $n$ number of positive cases, $N$ total of cases in each group

Table 2 Performance of epigenetic biomarkers for CRC's detection in tissue samples

\begin{tabular}{|c|c|c|c|c|c|c|}
\hline Gene/panels & Sensitivity (n/N) & Specificity (n/N) & AUC & PPV & NPV & Accuracy \\
\hline SEPT9 & $85.5(124 / 145)$ & $94.0(3 / 50)$ & 0.950 & 97.6 & 69.1 & 87.7 \\
\hline MGMT/SEPT9 & $93.8(136 / 145)$ & $82.0(9 / 50)$ & 0.964 & 93.8 & 82.0 & 90.8 \\
\hline MGMT/RASSFIA/SEPT9 & $96.6(140 / 145)$ & $74.0(13 / 50)$ & 0.970 & 91.5 & 88.1 & 90.8 \\
\hline
\end{tabular}

Sensitivity, Specificity, PPV and NPV values are represented in percentage

AUC Area under the curve, NPV negative predictive value, $P P V$ positive predictive value 
Table 4 Multivariable Cox regression analysis of disease specific survival

\begin{tabular}{|c|c|c|}
\hline Variable & Hazards ratio $(95 \% \mathrm{Cl})$ & $P$ value \\
\hline \multicolumn{3}{|l|}{ Colon and rectal samples $(\mathrm{N}=214)$} \\
\hline Age $(<60$ vs. $\geq 60)$ & $1.476(1.085-2.008)$ & 0.013 \\
\hline Stage (I/II vs. III) & $1.007(0.657-1.544)$ & 0.974 \\
\hline Stage (I/II vs. IV) & $1.862(1.295-2.677)$ & 0.001 \\
\hline Neoadjuvant treatment (no vs. yes) & $1.212(0.879-1.673)$ & 0.241 \\
\hline SEPT9 methylation (non-Hyper. vs. hypermethylated) & $0.673(0.469-0.965)$ & 0.031 \\
\hline \multicolumn{3}{|l|}{ Colon samples $(\mathrm{N}=110)$} \\
\hline $\operatorname{Age}(<60$ vs. $>=60)$ & $1.730(1.079-2.773)$ & 0.023 \\
\hline Stage (I/II vs. III) & $0.966(0.486-1.922)$ & 0.922 \\
\hline Stage (I/II vs. IV) & $1.454(0.859-2.463)$ & 0.164 \\
\hline Neoadjuvant treatment (no vs. yes) & $1.237(0.677-2.259)$ & 0.490 \\
\hline Tumor location (distal vs. proximal) & $1.879(1.174-3.007)$ & 0.009 \\
\hline SEPT9 methylation (non-Hyper. vs. hypermethylated) & $0.472(0.276-0.806)$ & 0.006 \\
\hline
\end{tabular}

95\% Cl 95\% confidence interval, CRC colorectal cancer, non-Hyper. non-hypermethylated

Italics values indicate statistically significant $(P<0.05)$

whereas the remainder genes showed significantly higher methylation levels in CRC, as reported by others [14, $15,17,30,31]$. Interestingly, SEPT9 and MGMT methylation levels were significantly lower in rectal cancer patients that underwent neoadjuvant treatment, which is in line with studies in which ionizing radiation exposure induced global hypomethylation [32, 33], including for colon cancer [34].

Regarding the use of gene promoter methylation as CRC biomarker, the best detection performance was disclosed by SEPT9 promoter methylation, followed by $M G M T$. Remarkably, SEPT9 validity estimates were similar to those of the trademark assays using SEPT9 methylation [12, 35], further confirming its biomarker potential. Nonetheless, MGMT displayed better performance $(77.2 \%$ sensitivity, $84.0 \%$ specificity) than previously reported (46-53\% sensitivity, $74-100 \%$ specificity), especially concerning sensitivity $[23,36]$. This might be due not only to differences in the population under study, but also differences in the methodological approaches. The same might apply to RASSF1A performance, which disclosed lower sensitivity (33.1\% vs. $81.0 \%$ ) but higher specificity (90.0\% vs. $51.0 \%$ ) [16], although the definition of the cutoff value might also significantly impact on

Table 5 Comparison of sensitivity and specificity of previously published panels with values obtained for this new panel

\begin{tabular}{|c|c|c|c|c|c|c|c|}
\hline Panels & Sensitivity (\%) & Specificity (\%) & AUC & Accuracy & Specimen type & Methods & References \\
\hline NGFR; SEPT9; TMEFF2 & NA & NA & 0.890 & NA & Tissue & qMSP & [39] \\
\hline NGFR; SEPT9; TMEFF2; bisDNA & & & 0.790 & NA & Plasma & & \\
\hline APC; MGMT; RASSF2A; Wif-1 & 86.5 & 92.1 & 0.927 & 89.2 & Plasma & MSP & [14] \\
\hline SFRP2; RASSF2 ${ }^{\mathrm{a}}$ & 75.0 & 89.4 & NA & NA & Stool & COBRA, Hi-SA & {$[40]$} \\
\hline BMP3; NDRG4; TFPI2; Vimentin & 78.0 & 85.0 & 0.880 & 91.2 & Stool & QUARTS & [41] \\
\hline HLTF; HPP1;MLH1 & NA & NA & NA & NA & Plasma & qMSP & [42] \\
\hline DCC; UNC5C & 82.0 & NA & NA & NA & Tissue & qMSP & [43] \\
\hline HPP1; MGMT; SFRP2 & 93.7 & 77.1 & NA & NA & Stool & MSP & {$[36]$} \\
\hline IGFBP3; miR137 & 95.5 & 90.5 & NA & 86.0 & Tissue & Bisulfite pyrosequencing & [44] \\
\hline CNRIP1; FBN1; INA; MAL; SNCA; SPG $20^{b}$ & 94.0 & 98.0 & 0.984 & 95.5 & Tissue & qMSP & [45] \\
\hline CMTM3; MDFI; SSTR2 & 81.0 & 91.0 & 0.920 & NA & Tissue & Bisulfite pyrosequencing & {$[46]$} \\
\hline MGMT; RASSFIA; SEPT9 & 96.6 & 74.0 & 0.970 & 90.8 & Tissue & $q M S P$ & - \\
\hline
\end{tabular}

COBRA combined bisulfite restriction analysis, Hi-SA high-sensitivity assay for bisulfite DNA, NA not available for the panel of genes, QuARTS quantitative allele-specific real-time target and signal amplification

a Also detects gastric cancer

b Co-methylation of two out of six genes 
performance. Nonetheless, a combination of these three gene promoters significantly improved diagnostic performance when compared with previously published panels (Table 5). In our experience, the use of gene promoter panels, usually comprising two to four genes, improves sensitivity, without compromising specificity, as previously demonstrated for prostate and breast cancers, especially when tested in liquid biopsies [37, 38]. Thus, the use of this gene panel might augment the performance of approved DNA methylation-based assays.

A potential downside of the use of DNA promoter methylation-based biomarkers for CRC detection is its potential association with a specific molecular subtype, namely MSI-H, which is due to defects in MMR pathway [3]. This system includes genes like $M L H 1, M S H 2$, MSH6, and PMS2 whose expression was screened by immunohistochemistry. Therefore, the loss of expression of any of these genes is considered indicative of defective MMR and, consequently, of MSI-H, whereas the remaining most likely represent MSI-L or MSS tumors. Using this strategy, only $3.7 \%$ of cases were disclosed as MSI$\mathrm{H}$, which is lower than reported in other series, especially those using direct MSI analysis [2, 3]. It is noteworthy, however, that in our series, almost $50 \%$ of tumors were localized in the rectum, whereas in most series rectal carcinoma represents less than $30 \%$ of CRC. Because rectal carcinoma usually displays a lower frequency of MSI-H cases, as verified in our series, the "over-representation" of tumors localized in the rectum might have decisively influenced the overall frequency. Curiously, CIMP cases were more frequent in the rectum $(11.5 \%$ vs. $5.6 \%)$, with an overall frequency of $8.5 \%$, which is close to the lower end of published series [2,3]. Owing to the lack of agreement on the best strategy to define a CRC case as CIMP, it is not possible, however, to perform direct comparisons. Nonetheless, it should be emphasized that excepting for $A P C$, no significant differences in gene promoter methylation levels were found among MSI-H, CIMP+ or CIN CRC molecular subtypes, nor among different pathological stages or tumor location, in our series. Thus, it may be concluded that the performance of the threegene promoter panel is likely to be homogeneous across molecular subtypes, and the same applies for primary tumor localization and pathological stage.

We also tested the prognostic value of the five genes promoter methylation status, as this might convey valuable information for clinical decision making. Remarkably, higher SEPT9 promoter methylation was independently associated with increased DSS in CRC, whereas no prognostic value was depicted for the remainder gene promoters. A meta-analysis that included 14 studies showed that MGMT methylation status was not associated with CRC prognosis [47], whereas RASSF1A promoter methylation has been associated with poor prognosis, although when assessed in plasma samples [30]. Furthermore, CIMP status also did not disclose prognostic value in our study, which is in accordance with a recent metaanalysis that found no significant effect of CIMP status in DFS or OS in 8 out of 11 and in 13 out of 19 studies previously published, respectively [48]. The same was reported regarding DSS, in 3 of 4 studies considering the classical CIMP panel [8]. Thus, globally, our results are by most of the published literature on the subject.

\section{Conclusions}

Our results indicate that MGMT/RASSF1A/SEPT9 gene promoter methylation panel accurately identifies CRC, irrespective of molecular subtype and may have a better performance than currently available epigeneticbased biomarkers. Nevertheless, the development of a clinically useful test derived from these results requires assessment of its performance in liquid biopsies, especially blood samples.

\section{Additional file}

Additional file 1: Table S1. General features of normal colon and rectum samples used for control purposes. Table S2. Primers sequence used for qMSP analysis.

\section{Abbreviations}

AJCC: American Joint Committee on Cancer; CIMP: CpG island methylator phenotype; CIMP-0: CIMP-negative; CIMP-H: CIMP-high; CIMP-L: CIMP-low; CIN chromosomal instability; CRC: colorectal cancer; CRN: non-malignant colorectal mucosas; FFPE: formalin-fixed paraffin-embedded; MMR: mismatch repair; MSI: microsatellite instability; MSI-H: MSI-high; MSS: microsatellite stable; ROC: receiver operator characteristics.

\section{Authors' contributions}

MF and FF performed DNA extraction and GMSP, analysed data and drafted the manuscript. SC assisted in the histopathological evaluation of tissue samples and in clinical follow-up data collection. FS and PL performed the FFPE sections for molecular analyses and the immunohistochemistry of all cases. FD and SS assisted in molecular analyses. LA assisted in the statistical analyses. LLS and JFV collected clinical follow-up data. RH performed histopathological evaluation of FFPE stained by H\&E. RH and $\mathrm{CJ}$ designed and supervised the study and revised the manuscript. All the authors read and approved the final manuscript.

\section{Author details \\ ${ }^{1}$ Cancer Biology \& Epigenetics Group-Research Center (CI-IPOP), Research Center-LAB 3, Portuguese Oncology Institute of Porto (IPO Porto), F Bdg, 1st Floor, Rua Dr António Bernardino de Almeida, 4200-072 Porto, Portugal. ${ }^{2}$ Departments of Pathology, Portuguese Oncology Institute of Porto (IPO Porto), Porto, Portugal. ${ }^{3}$ Departments of Epidemiology, Portuguese Oncol- ogy Institute of Porto (IPO Porto), Porto, Portugal. ${ }^{4}$ Departments of Surgical Oncology, Portuguese Oncology Institute of Porto (IPO Porto), Porto, Portugal. ${ }^{5}$ Department of Pathology and Molecular Immunology, Institute of Biomedi- cal Sciences Abel Salazar (ICBAS), University of Porto, Rua de Jorge Viterbo Ferreira n. 228 , 4050-313 Porto, Portugal.}

\section{Acknowledgements}

Not applicable. 


\section{Competing interests}

The authors declare that they have no competing interests.

\section{Availability of data and materials}

All data generated or analyzed during this study are included in this published article and its Additional information files.

\section{Consent for publication}

Not applicable.

\section{Ethics approval and consent to participate}

This study was approved by the institutional ethics committee (CES 120/015).

\section{Funding}

This work was supported by a grant from Research Center of Portuguese Oncology Institute of Porto (PI 74-Cl-IPOP-19-2016). SS is supported by a PhD fellowship IPO/ESTIMA-1 NORTE-01-0145-FEDER-000027.

\section{Publisher's Note}

Springer Nature remains neutral with regard to jurisdictional claims in published maps and institutional affiliations.

Received: 4 November 2017 Accepted: 16 February 2018

Published online: 27 February 2018

\section{References}

1. Ferlay J, Soerjomataram I, Dikshit R, Eser S, Mathers C, Rebelo M, Parkin DM, Forman D, Bray F. Cancer incidence and mortality worldwide: sources, methods and major patterns in GLOBOCAN 2012. Int J Cancer. 2015;136:E359-86.

2. Carethers JM, Jung BH. Genetics and genetic biomarkers in sporadic colorectal cancer. Gastroenterology. 2015;149(1177-1190):e1173.

3. Ogino S, Goel A. Molecular classification and correlates in colorectal cancer. J Mol Diagn. 2008;10:13-27.

4. Jass JR. Classification of colorectal cancer based on correlation of clinical, morphological and molecular features. Histopathology. 2007;50:113-30.

5. Kinzler KW, Vogelstein B. Lessons from hereditary colorectal cancer. Cell. 1996:87:159-70.

6. An B, Kondo Y, Okamoto Y, Shinjo K, Kanemitsu Y, Komori K, Hirai T, Sawaki A, Tajika M, Nakamura T, et al. Characteristic methylation profile in CpG island methylator phenotype-negative distal colorectal cancers. Int J Cancer. 2010:127:2095-105.

7. Weisenberger DJ, Siegmund KD, Campan M, Young J, Long TI, Faasse MA, Kang GH, Widschwendter M, Weener D, Buchanan D, et al. CpG island methylator phenotype underlies sporadic microsatellite instability and is tightly associated with BRAF mutation in colorectal cancer. Nat Genet. 2006:38:787-93.

8. Jia M, Gao X, Zhang Y, Hoffmeister M, Brenner H. Different definitions of CpG island methylator phenotype and outcomes of colorectal cancer: a systematic review. Clin Epigenet. 2016;8:25.

9. Estecio MRH, Issa JPJ. Dissecting DNA hypermethylation in cancer. FEBS Lett. 2011;585:2078-86.

10. Ogino S, Kawasaki T, Kirkner GJ, Loda M, Fuchs CS. CpG island methylator phenotype-low (CIMP-Low) in colorectal cancer: possible associations with male sex and KRAS mutations. J Mol Diagn. 2006;8:582-8.

11. Shen LL, Toyota M, Kondo Y, Lin E, Zhang L, Guo Y, Hernandez NS, Chen $\mathrm{XL}$, Ahmed S, Konishi K, et al. Integrated genetic and epigenetic analysis identifies three different subclasses of colon cancer. Proc Natl Acad Sci USA. 2007;104:18654-9.

12. Warren JD, Xiong W, Bunker AM, Vaughn CP, Furtado LV, Roberts WL, Fang JC, Samowitz WS, Heichman KA. Septin 9 methylated DNA is a sensitive and specific blood test for colorectal cancer. BMC Med. 2011:9:9.

13. Esteller M, Sparks A, Toyota M, Sanchez-Cespedes M, Capella G, Peinado MA, Gonzalez S, Tarafa G, Sidransky D, Meltzer SJ, et al. Analysis of adenomatous polyposis coli promoter hypermethylation in human cancer. Can Res. 2000:60:4366-71.

14. Lee BB, Lee EJ, Jung EH, Chun H-K, Chang DK, Song SY, Park J, Kim $\mathrm{D}-\mathrm{H}$. Aberrant methylation of APC, MGMT, RASSF2A, and Wif-1 genes in plasma as a biomarker for early detection of colorectal cancer. Clin Cancer Res Off J Am Assoc Cancer Res. 2009;15:6185-91.

15. Ogino S, Kawasaki T, Kirkner GJ, Suemoto Y, Meyerhardt JA, Fuchs CS. Molecular correlates with MGMT promoter methylation and silencing support CpG island methylator phenotype-low (CIMP-low) in colorectal cancer. Gut. 2007;56:1564-71

16. Sakamoto N, Terai T, Ajioka Y, Abe S, Kobayasi O, Hirai S, Hino O, Watanabe $\mathrm{H}$, Sato N, Shimoda T, Fujii H. Frequent hypermethylation of RASSF1A in early flat-type colorectal tumors. Oncogene. 2004;23:8900-7.

17. Wasserkort R, Kalmar A, Valcz G, Spisak S, Krispin M, Toth K, Tulassay Z, Sledziewski AZ, Molnar B. Aberrant septin 9 DNA methylation in colorectal cancer is restricted to a single CpG island. BMC Cancer. 2013;13:11.

18. Tóth K, Sipos F, Kalmár A, Patai ÁV, Wichmann B, Stoehr R, Golcher H, Schellerer V, Tulassay Z, Molnár B. Detection of methylated SEPT9 in plasma is a reliable screening method for both left- and right-sided colon cancers. PLoS ONE. 2012;7:e46000.

19. Miroglio A, Jammes H, Tost J, Ponger L, Gut IG, El Abdalaoui H, Coste J, Chaussade S, Arimondo PB, Lamarque D, Dandolo L. Specific hypomethylated CpGs at the IGF2 locus act as an epigenetic biomarker for familial adenomatous polyposis colorectal cancer. Epigenomics. 2010:2:365-75.

20. Cheng YW, Idrees K, Shattock R, Khan SA, Zeng ZS, Brennan CW, Paty P, Barany F. Loss of imprinting and marked gene elevation are 2 forms of aberrant IGF2 expression in colorectal cancer. Int J Cancer. 2010;127:568-77.

21. Baba Y, Nosho K, Shima K, Huttenhower C, Tanaka N, Hazra A, Giovannucci EL, Fuchs CS, Ogino S. Hypomethylation of the IGF2 DMR in colorectal tumors, detected by bisulfite pyrosequencing, Is Associated With Poor Prognosis. Gastroenterology. 2010;139:1855-64.

22. Church TR, Wandell M, Lofton-Day C, Mongin SJ, Burger M, Payne SR, Castaños-Vélez E, Blumenstein BA, Rösch T, Osborn N, et al. Prospective evaluation of methylated SEPT9 in plasma for detection of asymptomatic colorectal cancer. Gut. 2014;63:317-25.

23. Shen LL, Kondo Y, Rosner GL, Xiao LC, Hernandez NS, Vilaythong J, Houlihan PS, Krouse RS, Prasad AR, Einspahr JG, et al. MGMT promoter methylation and field defect in sporadic colorectal cancer. J Nat Cancer Inst. 2005:97:1330-8.

24. Pinheiro M, Ahlquist T, Danielsen SA, Lind GE, Veiga I, Pinto C, Costa V Afonso L, Sousa O, Fragoso M, et al. Colorectal carcinomas with microsatellite instability display a different pattern of target gene mutations according to large bowel site of origin. BMC Cancer. 2010;10:587.

25. Ramalho-Carvalho J, Pires M, Lisboa S, Graca I, Rocha P, Barros-Silva JD, Savva-Bordalo J, Mauricio J, Resende M, Teixeira MR, et al. Altered expression of MGMT in high-grade gliomas results from the combined effect of epigenetic and genetic aberrations. PLoS ONE. 2013;8:e58206.

26. Toyota M, Ahuja N, Ohe-Toyota M, Herman JG, Baylin SB, Issa JP. CpG island methylator phenotype in colorectal cancer. Proc Natl Acad Sci USA. 1999;96:8681-6.

27. Inadomi JM. Screening for Colorectal Neoplasia. N Engl J Med. 2017:376:1599-600.

28. Ned RM, Melillo S, Marrone M. Fecal DNA testing for colorectal cancer screening: the ColoSure test. PLoS currents. 2011;3:RRN1220.

29. Gyparaki MT, Basdra EK, Papavassiliou AG. DNA methylation biomarkers as diagnostic and prognostic tools in colorectal cancer. J Mol Med Jmm. 2013;91:1249-56

30. Matthaios D, Balgkouranidou I, Karayiannakis A, Bolanaki H, Xenidis N, Amarantidis K, Chelis L, Romanidis K, Chatzaki A, Lianidou E, et al. Methylation status of the APC and RASSF1A promoter in cell-free circulating DNA and its prognostic role in patients with colorectal cancer. Oncol Lett. 2016;12:748-56.

31. Song L, Li Y. SEPT9: a specific circulating biomarker for colorectal cancer. Adv Clin Chem. 2015;72:171-204.

32. Hofstetter B, Niemierko A, Forrer C, Benhattar J, Albertini V, Pruschy M, Bosman FT, Catapano CV, Ciernik IF. Impact of genomic methylation on radiation sensitivity of colorectal carcinoma. Int J Radiat Oncol Biol Phys. 2010;76:1512-9

33. Kim JG, Park MT, Heo K, Yang KM, Yi JM. Epigenetics meets radiation biology as a new approach in cancer treatment. Int J Mol Sci. 2013;14:15059-73.

34. Bae JH, Kim JG, Heo K, Yang K, Kim TO, Yi JM. Identification of radiationinduced aberrant hypomethylation in colon cancer. BMC Genomics. 2015;16:56 
35. Wu D, Zhou G, Jin P, Zhu J, Li S, Wu Q, Wang G, Sheng J, Wang J, Song $L$, et al. Detection of colorectal cancer using a simplified SEPT9 gene methylation assay is a reliable method for opportunistic screening. J Mol Diagn. 2016;18:535-45.

36. Huang ZH, Li LH, Yang F, Wang JF. Detection of aberrant methylation in fecal DNA as a molecular screening tool for colorectal cancer and precancerous lesions. World J Gastroenterol. 2007;13:950-4.

37. Jeronimo C, Henrique R. Epigenetic biomarkers in urological tumors: a systematic review. Cancer Lett. 2014:342:264-74.

38. Costa-Pinheiro P, Montezuma D, Henrique R, Jeronimo C. Diagnostic and prognostic epigenetic biomarkers in cancer. Epigenomics. 2015;7:1003-15.

39. Lofton-Day C, Model F, DeVos T, Tetzner R, Distler J, Schuster M, Song XL, Lesche R, Liebenberg V, Ebert M, et al. DNA methylation biomarkers for blood-based colorectal cancer screening. Clin Chem. 2008;54:414-23.

40. Nagasaka T, Tanaka N, Cullings HM, Sun DS, Sasamoto H, Uchida T, Koi M, Nishida N, Naomoto Y, Boland CR, et al. Analysis of fecal DNA methylation to detect gastrointestinal neoplasia. J Nat Cancer Inst. 2009;101:1244-58.

41. Ahlquist DA, Zou HZ, Domanico M, Mahoney DW, Yab TC, Taylor WR, Butz ML, Thibodeau SN, Rabeneck L, Paszat LF, et al. Next-generation stool DNA test accurately detects colorectal cancer and large adenomas. Gastroenterology. 2012;142:248-56.

42. Wallner M, Herbst A, Behrens A, Crispin A, Stieber P, Goke B, Lamerz R, Kolligs FT. Methylation of serum DNA is an independent prognostic marker in colorectal cancer. Clin Cancer Res. 2006;12:7347-52.
43. Hibi K, Mizukami H, Shirahata A, Goto T, Sakata M, Sanada Y. Aberrant methylation of the Netrin-1 receptor genes UNC5C and DCC detected in advanced colorectal cancer. World J Surg. 2009;33:1053-7.

44. Perez-Carbonell L, Balaguer F, Toiyama Y, Egoavil C, Rojas E, Guarinos C, Andreu M, Llor X, Castells A, Jover R, et al. IGFBP3 methylation is a novel diagnostic and predictive biomarker in colorectal cancer. PLOS ONE. 2014;9:11.

45. Lind GE, Danielsen SA, Ahlquist T, Merok MA, Andresen K, Skotheim Rl, Hektoen M, Rognum TO, Meling Gl, Hoff G, et al. Identification of an epigenetic biomarker panel with high sensitivity and specificity for colorectal cancer and adenomas. Mol Cancer. 2011;10:15.

46. Li JY, Chen C, Bi XE, Zhou CC, Huang T, Ni C, Yang P, Chen S, Ye M, Duan SW. DNA methylation of CMTM3, SSTR2, and MDFI genes in colorectal cancer. Gene. 2017;630:1-7.

47. Li Y, Lyu Z, Zhao L, Cheng H, Zhu D, Gao Y, Shang X, Shi H. Prognostic value of MGMT methylation in colorectal cancer: a meta-analysis and literature review. Tumour Biol. 2015;36:1595-601.

48. Juo YY, Johnston FM, Zhang DY, Juo HH, Wang H, Pappou EP, Yu T, Easwaran H, Baylin S, van Engeland M, Ahuja N. Prognostic value of CpG island methylator phenotype among colorectal cancer patients: a systematic review and meta-analysis. Ann Oncol. 2014;25:2314-27.

\section{Submit your next manuscript to BioMed Central and we will help you at every step:}

- We accept pre-submission inquiries

- Our selector tool helps you to find the most relevant journal

- We provide round the clock customer support

- Convenient online submission

- Thorough peer review

- Inclusion in PubMed and all major indexing services

- Maximum visibility for your research

Submit your manuscript at www.biomedcentral.com/submit 